\title{
Measuring radiofrequency fields in NMR spectroscopy using offset-dependent nutation profiles
}

\author{
Ahallya Jaladeep ${ }^{1}$, Claris Niya Varghese ${ }^{1}$, Ashok Sekhar ${ }^{*}$ \\ Molecular Biophysics Unit, Indian Institute of Science, Bangalore 560 012, India
}

\section{A R T I C L E I N F O}

\section{Article history:}

Received 26 May 2021

Revised 2 July 2021

Accepted 4 July 2021

Available online 6 July 2021

\section{Keywords:}

NMR spectroscopy

Radiofrequency field strength

Nutation

CEST

Relaxation dispersion

$\mathrm{RF}$ inhomogeneity

\begin{abstract}
A B S T R A C T
The application of NMR spectroscopy for studying molecular and reaction dynamics relies crucially on the measurement of the magnitude of radiofrequency (RF) fields that are used to nutate or lock the nuclear magnetization. Here, we report a method for measuring RF field amplitudes that leverages the intrinsic modulations observed in offset-dependent NMR nutation profiles of small molecules. Such nutation profiles are exquisitely sensitive to the magnitude of the $\mathrm{RF}$ field, and $\mathrm{B}_{1}$ values ranging from 1 to $2000 \mathrm{~Hz}$, as well the inhomogeneity in $\mathrm{B}_{1}$ distributions, can be determined with high accuracy and precision using this approach. In order to measure $B_{1}$ fields associated with NMR experiments carried out on protein or nucleic acids, where these modulations are obscured by the large transverse relaxation rate constants of the analyte, our approach can be used in conjunction with a suitable external small molecule standard, expanding the scope of the method for large biomolecules.
\end{abstract}

(c) 2021 Elsevier Inc. All rights reserved.

\section{Introduction}

The NMR toolbox for characterizing molecular dynamics has diversified considerably in the last two decades with the introduction of methods such as chemical exchange saturation transfer (CEST) and relaxation dispersion (RD) [1-12]. This has led to the detection and structural characterization of a number of sparsely populated biomolecular conformations implicated in enzyme catalysis [13,14], molecular recognition [15-18] and protein folding $[19,20]$, as well as in aggregation [21,22] and disease [23-25]. While $R_{1 \rho}$ RD experiments have identified transient Hoogsteen base pairs in duplex DNA [26,27], mechanisms underlying base misincorporation during DNA replication [14,28] and invisible excited states of HIV-1 TAR RNA [29], CEST has been employed to visualize higher energy functional conformations of Abl kinase [25], superoxide dismutase [23,24] and the fluoride riboswitch [17], as well as to define the conformational selection mechanism underlying Hsp70 chaperone-substrate interactions [30]. Moreover, the populations and lifetimes of transiently populated reaction intermediates in organic and metallorganic chemistry have recently been estimated with the CEST approach [31-35].

\footnotetext{
* Corresponding author at: Molecular Biophysics Unit, Indian Institute of Science Bangalore, Bengaluru 560 012, Karnataka, India.

E-mail address: ashoksekhar@iisc.ac.in (A. Sekhar).

1 Both these authors contributed equally to this work.
}

In both CEST and $\mathrm{R}_{1 \rho}$ RD experiments, an accurate measurement of the radiofrequency (RF) field strength is essential for quantifying thermodynamic, kinetic and structural parameters of the conformational exchange event $[11,36,37]$. Over the years, a number of methods have been developed for determining the amplitude of the $B_{1}$ field, beginning with the sideband strategy outlined by Bloch [38] and demonstrated by Anderson in 1956 [39]. With the advent of pulsed NMR, nutation resulting from onresonance irradiation was proposed as an efficient method for calibrating the applied RF field $[40,41]$. In heteronuclear NMR involving ${ }^{15} \mathrm{~N}$ or ${ }^{13} \mathrm{C}$ isotope-labeled samples, the magnitude of the $\mathrm{B}_{1}$ field can be measured from the residual splitting observed in a multiplet pattern when the decoupling field is applied offresonance [36,42-44]. While this method is ideal for $\mathrm{B}_{1}$ amplitudes comparable to or larger than the heteronuclear coupling constant (90-150 Hz), much smaller $B_{1}$ fields are routinely used in CEST experiments.

The current method for measuring weak $B_{1}$ fields proposed by Guenneugues et al. [45] is a variation of the nutation experiment $[41,46]$ where the RF field of desired strength is applied on $z-$ magnetization for a systematically incremented time duration. Transverse components of magnetization are subsequently dephased with a gradient and the z-component is quantified through a read-pulse. Fourier transformation of this timedependent signal provides both the amplitude and the probability distribution of the $B_{1}$ field across the sample. While this approach has been successful over a broad range of $B_{1}$ field strengths, it is 
challenging to use when measuring small RF fields of the order of 1-10 $\mathrm{Hz}$ using resonances of biomolecules such as proteins or nucleic acids. This is because of the need to place the RF transmitter on-resonance to within a value much smaller than the magnitude of the $B_{1}$ field, or alternatively to treat the chemical shift offset as a fitting parameter while modeling intensities in the nutation spectrum.

In this manuscript, we report a method for measuring radiofrequency (RF) fields that makes use of modulations observed in the constant-time offset-dependent nutation profile of $z$ magnetization (CONDENZ) under the influence of RF radiation. This method enables the precise and accurate calibration of RF fields and is particularly useful for weak RF fields of the order of 1$10 \mathrm{~Hz}$ employed in CEST experiments. In addition, the CONDENZ approach provides a robust estimate of the inhomogeneity in the $B_{1}$ field that is required for the quantitative analysis of CEST and $\mathrm{R}_{1 \rho} \mathrm{RD}$ profiles.

\section{Materials and methods}

\subsection{Sample preparation}

An NMR sample of $100 \mathrm{mM}$ unlabeled sucrose was prepared in $25 \mathrm{mM}$ Tris buffer at pH 7 with $90 \% \mathrm{D}_{2} \mathrm{O} / 10 \% \mathrm{H}_{2} \mathrm{O}, 0.03 \% \mathrm{NaN}_{3}$ and $1 \mathrm{mM}$ DSS. This sucrose sample was used for all data collection for the ${ }^{13} \mathrm{C}$ CONDENZ profiles shown in Fig. 1 and Figure S1. ${ }^{13} \mathrm{C}$ RF field strengths were also measured on a sample containing a mixture of $100 \mathrm{mM}$ unlabeled sucrose, $1 \mathrm{mM}$ methyl- ${ }^{13} \mathrm{C}$ $\alpha$-ketobutyric acid and $50 \mathrm{mM}$ benzaldehyde prepared in $25 \mathrm{mM}$ Tris buffer at pH 7 with $90 \% \mathrm{D}_{2} \mathrm{O} / 10 \% \mathrm{H}_{2} \mathrm{O}, 0.03 \% \mathrm{NaN}_{3}$ and $1 \mathrm{mM}$ DSS.

RF fields on the ${ }^{15} \mathrm{~N}$ nucleus were measured on two different NMR samples. The first one contained $1 \mathrm{mM}{ }^{15} \mathrm{~N}^{\varepsilon}$-labeled tryptophan and $0.8 \mathrm{mM} \mathrm{U}-{ }^{15} \mathrm{~N}$ ubiquitin, prepared in $25 \mathrm{mM}$ sodium phosphate buffer at $\mathrm{pH} 7$ with $25 \mathrm{mM} \mathrm{NaCl}, 1 \mathrm{mM}$ EDTA, $0.03 \% \mathrm{NaN}_{3}$ and $2.5 \% \mathrm{~d}_{6}$-DMSO. The second one was used as an external standard for ${ }^{15} \mathrm{~N} \mathrm{~B}_{1}$ calibration and contained $1 \mathrm{mM}{ }^{15} \mathrm{~N}^{\varepsilon}$-labelled tryptophan prepared in the same buffer as the ${ }^{15} \mathrm{~N}^{\varepsilon}$-labeled tryptophan/U- ${ }^{15} \mathrm{~N}$ ubiquitin sample mentioned above. The aprotic solvent $d_{6}$-DMSO served as the lock solvent in both samples in order to eliminate artifacts from H/D solvent exchange [47].

$\mathrm{U}-{ }^{15} \mathrm{~N}$ ubiquitin was overexpressed as a construct without any purification tag in Escherichia coli (E.coli) BL21(DE3) cells grown in 2xM9 media [48] with $1 \mathrm{~g} / \mathrm{L}$ of ${ }^{15} \mathrm{NH}_{4} \mathrm{Cl}$ as the sole nitrogen source. Cell pellets were lysed by sonication and purified as described earlier [49]. Briefly, the $\mathrm{pH}$ of the clarified cell lysate was adjusted to 4.5 by drop-wise addition of acetic acid to precipitate many of the endogenous E.coli proteins. The mixture was centrifuged at $15000 \mathrm{rpm}$ for $1 \mathrm{~h}$ and the supernatant was dialysed against $50 \mathrm{mM}$ acetic acid/sodium acetate buffer at $\mathrm{pH}$ 4.5. Ubiquitin was then loaded on an SP-sepharose cation exchange column and eluted with a $0-500 \mathrm{mM} \mathrm{NaCl}$ gradient in the same buffer. Ubiquitin eluted at an $\mathrm{NaCl}$ concentration of $260 \mathrm{mM}$. Fractions containing ubiquitin were pooled, concentrated and loaded on a 16/600 Superdex 75 size exclusion chromatographic column for subsequent purification. Pure fractions were pooled, concentrated, aliquoted, flash frozen and stored at $-80{ }^{\circ} \mathrm{C}$.

\subsection{NMR spectroscopy}

All NMR experiments were performed at $25^{\circ} \mathrm{C}$ on a $700 \mathrm{MHz}$ Bruker spectrometer equipped with a room temperature triple resonance single-axis gradient TXI probe.

\subsection{CONDENZ measurements}

CONDENZ data were acquired in pseudo-2D mode using the pulse sequence shown in Fig. 2. In each dataset, the RF field is positioned at a specific offset value and a $1 \mathrm{D}$ spectrum is acquired that typically contains only one peak at the chemical shift of the proton which is scalar coupled to the target ${ }^{13} \mathrm{C}$ $\left({ }^{15} \mathrm{~N}\right)$ nucleus. The offset position of the RF field is then swept through the ${ }^{13} \mathrm{C}\left({ }^{15} \mathrm{~N}\right)$ chemical shift of the peak of interest, generating one 1D spectrum corresponding to each offset. Every pseudo-2D CONDENZ dataset also contains a reference 1D spectrum acquired with the same pulse sequence, but with $t_{n u t}$ set to 0 . The sweep range and the offset step-size depend on the magnitude of the applied RF field and are tabulated in Table S1. For example, for a $10 \mathrm{~Hz} \mathrm{~B}_{1}$ field, a pseudo-2D dataset containing 92 1D spectra was generated by sweeping from -90 to $90 \mathrm{~Hz}$ using a uniform spacing between successive offsets of $2 \mathrm{~Hz}$. Signal averaging over 16 transients for each 1D spectrum, corresponding to an average acquisition time of $\sim 40 \mathrm{~min}$ per $\mathrm{B}_{1}$ field, usually gave sufficient signal-to-noise (SNR) for quantitative analysis. The relaxation delay used in all CONDENZ measurements was $1.5 \mathrm{~s}$.

\subsection{Analysis of CONDENZ datasets}

Pseudo 2D CONDENZ data were processed and analysed using the Bruker Topspin version 4.0.7 software package. 2D datasets were first split into individual 1D spectra using the command 'splitser', following which the peaks in 1D spectra acquired at different offsets were picked using the automated Topspin routine 'pps'. The intensities in the offset-dependent 1D spectra (I) were plotted as a ratio against the corresponding intensity in the reference spectrum $\left(t_{n u t}=0, \mathrm{I}_{0}\right)$ as a function of the offset at which the RF field is applied to generate CONDENZ profiles. Offsets were measured as differences from the onresonance chemical shift of the target ${ }^{13} \mathrm{C}$ nucleus, which was set to $0 \mathrm{~Hz}$.

The errors in the intensity values were obtained using the 'SINO' routine. First, the regions corresponding to the signal and to noise in each individual 1D spectrum are selected. SINO is then used to determine the SNR for each spectrum in the CONDENZ dataset. The noise, which is used as the error estimate in intensity, is then evaluated from the offset-specific intensity and SNR values. Typically, $0.05 \mathrm{ppm}$ surrounding the peak of interest and $0.5 \mathrm{ppm}$ in the region $0.5-1.0 \mathrm{ppm}$ were chosen as the signal and noise regions in SINO. For offset values near resonance, the intensity is close to 0 . The error value assigned in such cases is the average of the errors estimated from spectra belonging to the same pseudo-2D dataset where the intensity is sufficiently large to quantify the error.

\section{Results and discussion}

\subsection{Intensity modulations observed in offset-dependent nutation profiles}

Fig. 1A and Figure S1 show CONDENZ profiles of the anomeric ${ }^{13} \mathrm{C}$ magnetization of the glucose ring in sucrose (referred to hereafter as the sucrose anomeric carbon) for $\mathrm{B}_{1}$ fields ranging from 1 $2000 \mathrm{~Hz}$. Nutation data were acquired using a selective 1D-based ${ }^{13} \mathrm{C}$-CEST pulse sequence shown in Fig. 2 . In this sequence, ${ }^{13} \mathrm{C} z-$ magnetization of the anomeric sucrose carbon $\left(C_{z}\right)$ is created from the single-bonded anomeric ${ }^{1} \mathrm{H}$ magnetization by transfer via a selective J cross-polarization module [50,51] (Fig. 2). $C_{z}$ is then subjected to a $B_{1}$ field applied at a specific ${ }^{13} \mathrm{C}$ offset for a constant 
A) $\quad 0.98 \pm 0.01 \mathrm{~Hz}$
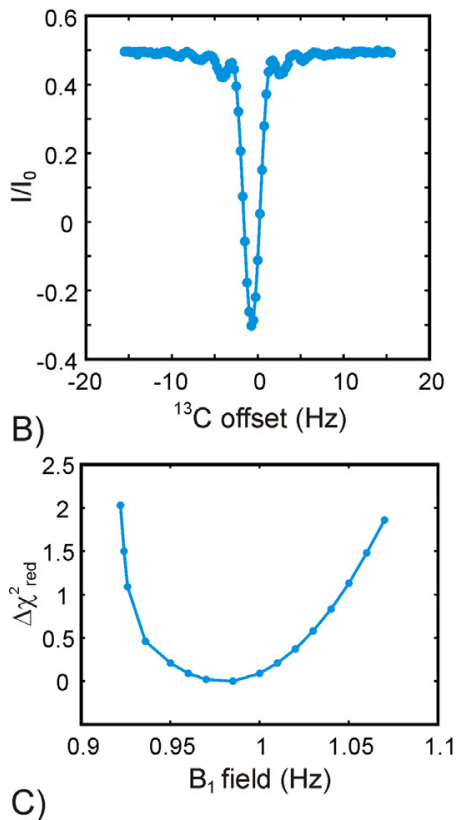

C)

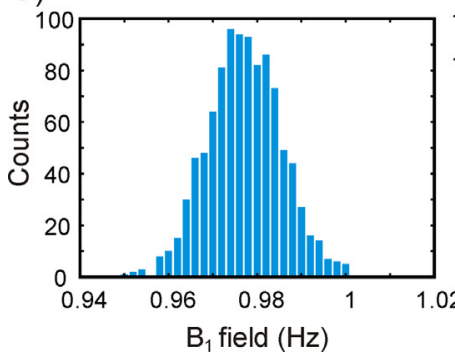

$9.94 \pm 0.01 \mathrm{~Hz}$
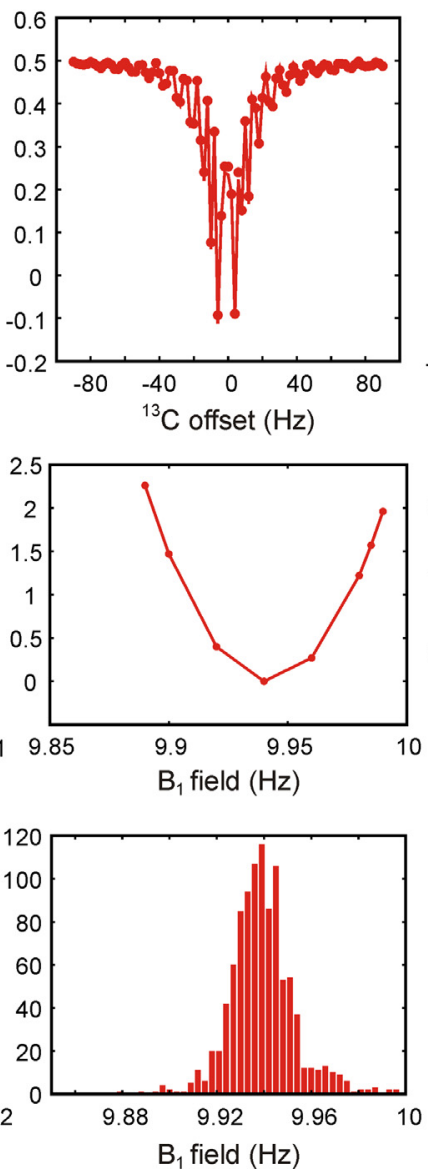

$97.20 \pm 0.04 \mathrm{~Hz}$
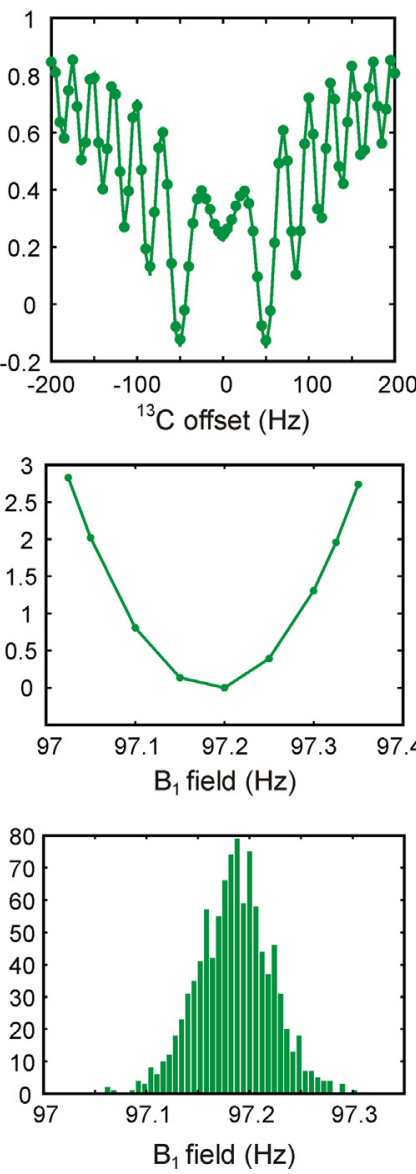

$973.4 \pm 0.4 \mathrm{~Hz}$
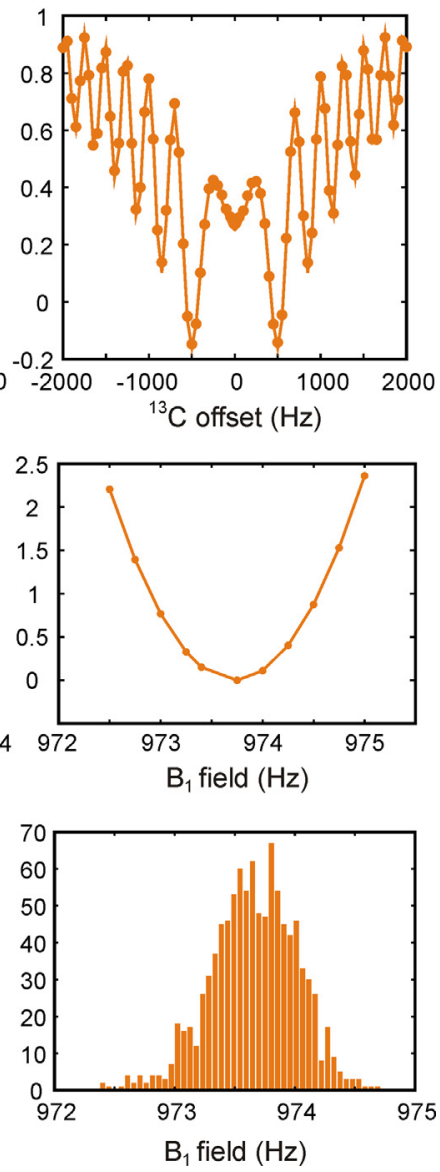

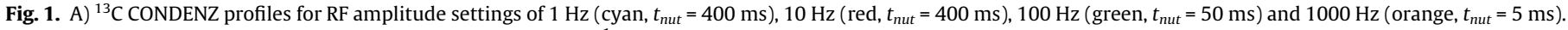

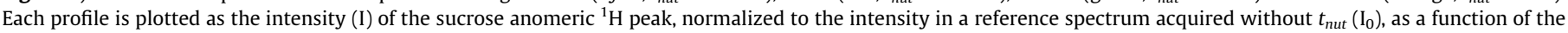

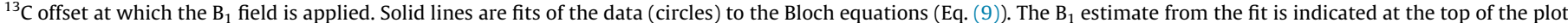

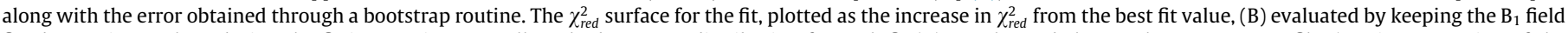

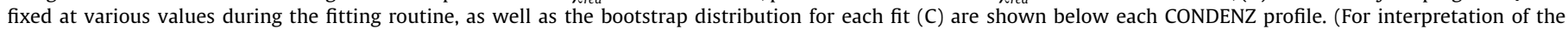
references to colour in this figure legend, the reader is referred to the web version of this article.)

nutation time $t_{n u t}$, during which scalar coupled protons are decoupled via a DIPSI-2 [52] composite pulse decoupling train. The ${ }^{13} \mathrm{C} \mathrm{z}-$ magnetization at the end of $t_{\text {nut }}$ is then transferred back to ${ }^{1} \mathrm{H}$ using the same selective Hartmann-Hahn transfer for detection. A reference spectrum is acquired for each value of the $B_{1}$ field using the same pulse sequence but where the nutation period is absent. CONDENZ profiles in Fig. 1A graph the intensity of the sucrose anomeric ${ }^{1} \mathrm{H}$ peak (I) as a ratio against the intensity of the target peak in the reference spectrum $\left(\mathrm{I}_{0}\right)$ as a function of the ${ }^{13} \mathrm{C}$ offset at which the $B_{1}$ field is applied.

\subsection{Theoretical basis for modulations seen in CONDENZ profiles}

The presence of offset-dependent modulations observed in CONDENZ profiles can be explained through an analysis of the Bloch equations. For a single-spin- $1 / 2$ system, the Bloch equations in the rotating frame for the three components of magnetization $\left(M_{x}, M_{y}\right.$ and $\left.M_{z}\right)$ in the presence of a RF field of amplitude $\omega_{1}$ ( $\mathrm{rad} / \mathrm{s})$ applied along the $\mathrm{x}$-axis are given by [55]:

$\frac{d \vec{M}}{d t}=R \vec{M}$

where $\vec{M}=\left[\begin{array}{llll}E & M_{x} & M_{y} & M_{z}\end{array}\right]^{T}$ ( $T$ being the transpose operation), E is identity and
$R=\left[\begin{array}{cccc}0 & 0 & 0 & 0 \\ 0 & -R_{2} & -\Delta & 0 \\ 0 & \Delta & -R_{2} & -\omega_{1} \\ R_{1} M_{0} & 0 & \omega_{1} & -R_{1}\end{array}\right]$

$\Delta$ is the chemical shift offset ( $\mathrm{rad} / \mathrm{s}), \mathrm{R}_{1}$ and $\mathrm{R}_{2}$ are longitudinal and transverse relaxation rate constants respectively, and $\mathrm{M}_{0}$ is the equilibrium magnetization. The formal solution to this set of coupled differential equations is:

$\vec{M}(t)=e^{R t} \vec{M}(0)$

For small molecules, where relaxation rates are slow compared to $\Delta$ or $\omega_{1}$, we can neglect relaxation and the simplified equations of motion in the rotating frame become:

$\frac{d M_{x}(t)}{d t}=-\Delta M_{y}(t)$
$\frac{d M_{y}(t)}{d t}=\Delta M_{x}(t)-\omega_{1} M_{z}(t)$
$\frac{d M_{z}(t)}{d t}=\omega_{1} M_{y}(t)$.

Assuming initial conditions of:

$M_{x}(0)=M_{y}(0)=0$,

$M_{z}(0)=M_{0}$ 

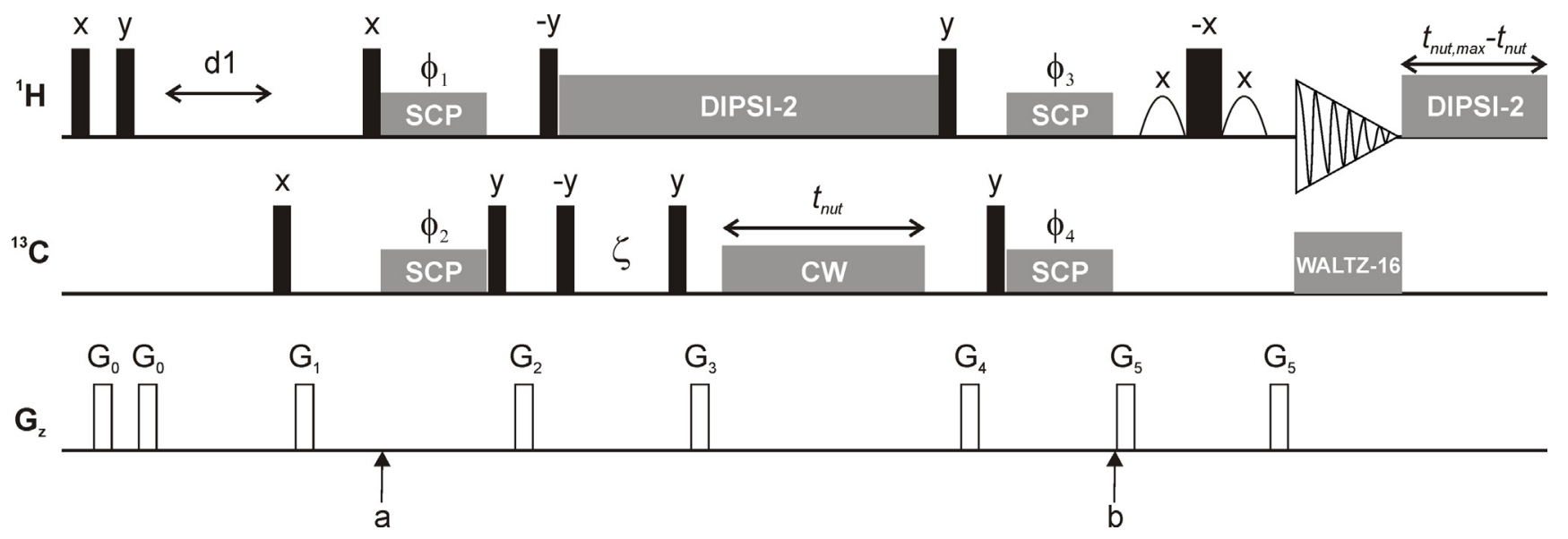

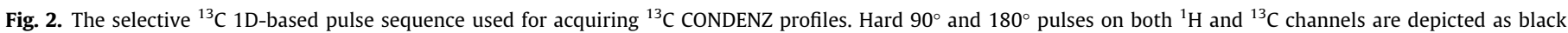

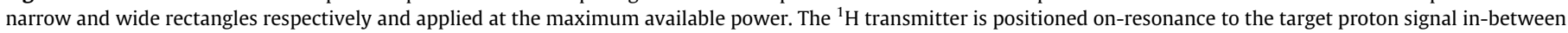

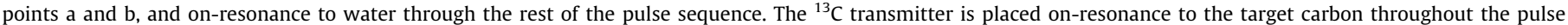

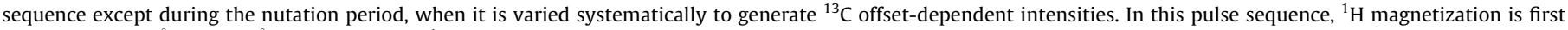

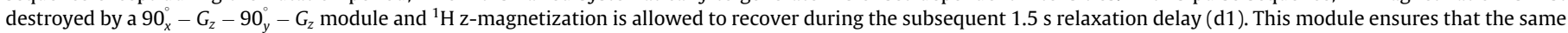

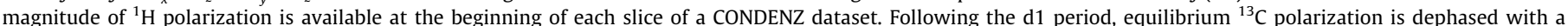

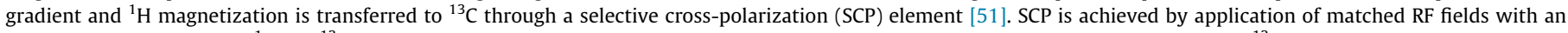

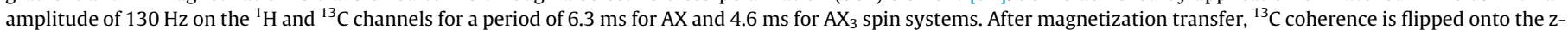

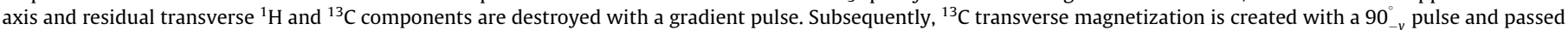

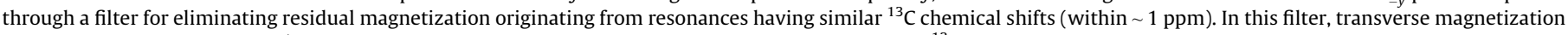

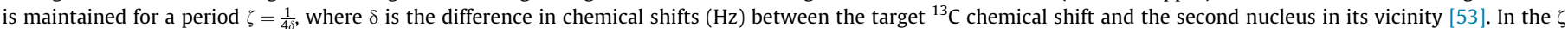

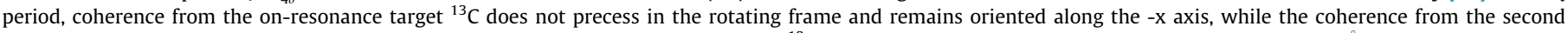

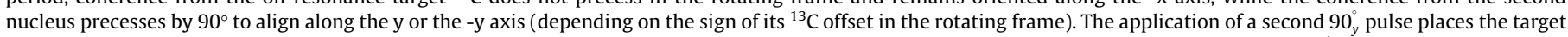

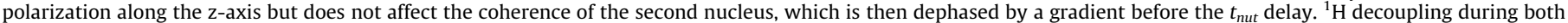

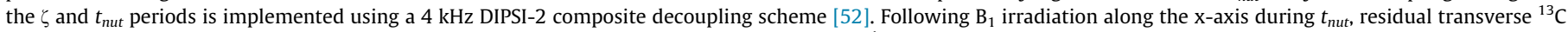

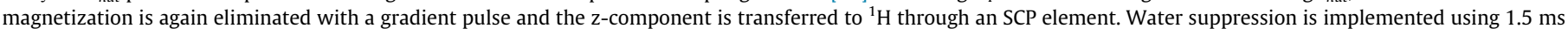

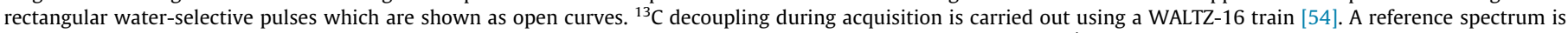

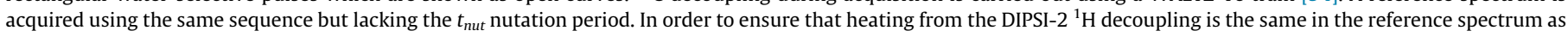

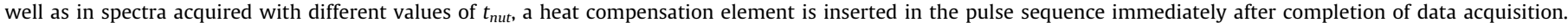

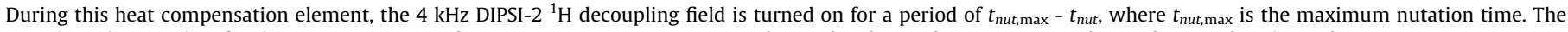

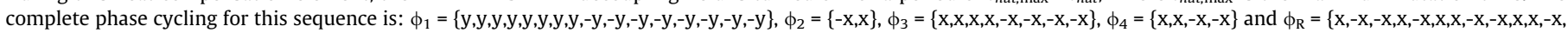

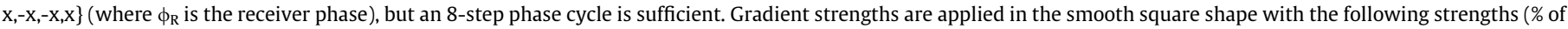

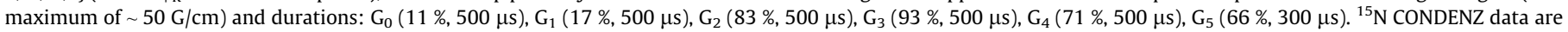

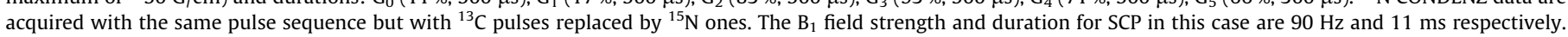

the analytical solution for $M_{z}\left(t_{n u t}\right)$ is given by:

$\frac{M_{z}\left(t_{n u t}\right)}{M_{0}}=1-\left[\frac{\omega_{1}^{2} t_{n u t}^{2}}{2}\left(\frac{\sin \phi}{\phi}\right)^{2}\right]$,

where

$\phi=\frac{\sqrt{\Delta^{2}+\omega_{1}^{2}} t_{\text {nut }}}{2}$

The trajectory of z-magnetization as a function of the offset $\Delta$ is thus a squared sinc function, matching experimental observations shown in Fig. 1A. The argument of the sinc function depends on the variable chemical shift offset $\Delta$, as well as the values of the nutation time and the amplitude of the applied radiofrequency field, which are held constant while acquiring nutation profiles.

\subsection{CONDENZ profiles are exceptionally sensitive to the $B_{1}$ field strength}

Interestingly, simulations of the Bloch equations (Fig. 3) reveal that these nutation profiles are exquisitely sensitive to the magnitude of the RF field; for example, profiles simulated with $B_{1}$ values of 3 and $3.2 \mathrm{~Hz}$ are visibly different. Therefore, we reasoned that experimentally acquired nutation profiles should enable us to measure the corresponding $\mathrm{B}_{1}$ field with high precision.

Accordingly, we modeled nutation profiles shown in Fig. $1 \mathrm{~A}$ using the Bloch equations, assuming the anomeric ${ }^{13} \mathrm{C}$ to be a single-spin- $1 / 2$ system. Longitudinal $\left(R_{1}\right)$ and transverse $\left(R_{2}\right)$ relax- ation rates of the anomeric carbon, as well as its chemical shift and the $B_{1}$ field were treated as fitting parameters (Table S2). The RF amplitude was assumed to follow a Gaussian distribution with its standard deviation defined as the $\mathrm{B}_{1}$ inhomogeneity $\left(I_{B 1}\right)$ [56]. $\mathrm{N}$ (typically, $\mathrm{N}=11$ ) samples of the $\mathrm{B}_{1}$ field $\left(\mathrm{B}_{1 \mathrm{~s}}\right)$ were drawn from this distribution within the range $\left[\left(B_{1}-2 I_{B 1}\right),\left(B_{1}+2 I_{B 1}\right)\right]$ and the probabilities of their occurrence were determined from the equation for the Gaussian probability distribution function as:

$P\left(B_{1 s}\right)=\frac{1}{\sqrt{2 \pi I_{B 1}^{2}}} \exp \left(-\frac{\left(B_{1 s}-B_{1}\right)^{2}}{2 I_{B 1}^{2}}\right)$

The formal solution of the Bloch equations depends on the particular value of $B_{15}$, so that the overall magnetization at the end of the time-evolution is a weighted sum of the individual evolutions, with the weights given by $\mathrm{P}\left(\mathrm{B}_{1 \mathrm{~s}}\right)$ as:

$\vec{M}(t)=\sum_{N} P\left(B_{1 S}\right) M_{S}(t)=\sum_{N} P\left(B_{1 S}\right) e^{R\left(B_{1 S}\right) t} \vec{M}(0)$

where the evolution matrix $\mathrm{R}$ now is a function of the particular sampled value of $B_{1 s}$.

CONDENZ profiles can be fit very well using the single-spin- $1 / 2$ Bloch equations described by Eq. (9) to recover the magnitude of the RF field (Fig. 1A, Fig. S1). $\chi_{\text {red }}^{2}$ surfaces as a function of $B_{1}$ are very steep (Fig. 1B, Fig. S1), demonstrating the robustness of the $B_{1}$ values obtained from data fitting. In order to determine the precision of the measured $B_{1}$ values, we estimated errors using a bootstrap algorithm [57], where 1000 bootstrapped datasets for each $B_{1}$ 

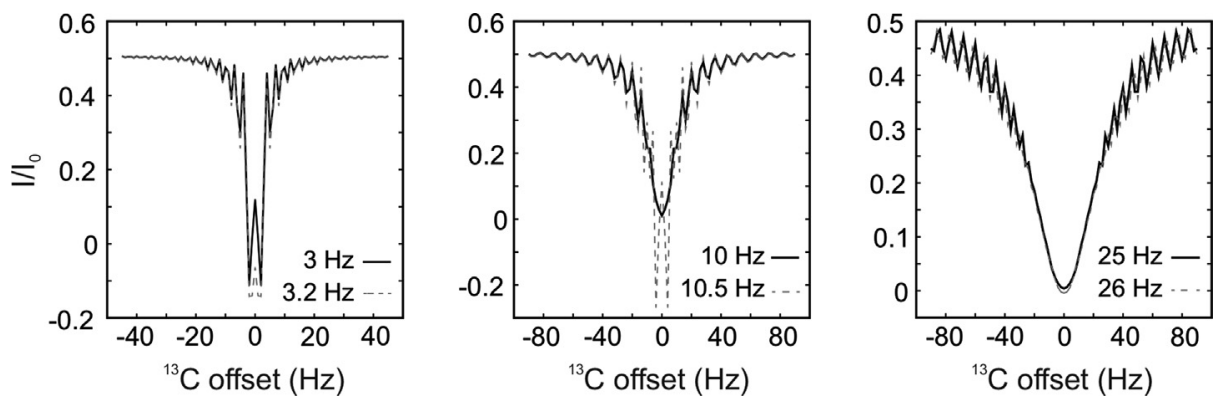

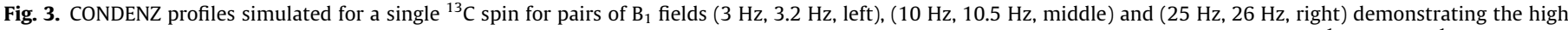

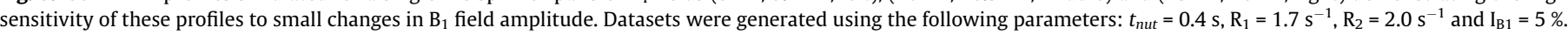

field were constructed from the nutation profile by random sampling with replacement and fit to the Bloch equations. The resulting bootstrapped $\mathrm{B}_{1}$ distributions (Fig. $1 \mathrm{C}$, Fig. S1, Table 1) are narrow with standard deviations ranging from $0.02-1 \%$ of the measured $B_{1}$ value, clearly showing that highly precise $B_{1}$ measurements can be made from the modeling of offset-dependent nutation curves.

\subsection{Evaluating the accuracy of $B_{1}$ amplitudes extracted from CONDENZ profiles}

As the next step, we evaluated the data for the presence of systematic errors that could bias the $\mathrm{B}_{1}$ estimates. First, we experimentally measured the magnitude of $B_{1}$ using a second method proposed by Guenneugues et al $[45,58]$, in which magnetization is nutated by an external $B_{1}$ field applied on-resonance to the peak of interest for a variable nutation time. The time-dependent signal intensity is then Fourier transformed to determine the $B_{1}$ field. $B_{1}$ values obtained from CONDENZ profiles and the on-resonance nutation method agree very well with an $R^{2}$ value of 0.99 (Fig. 4, Table 1), demonstrating the reliability of the RF field measurements that can be made with the CONDENZ method.

Second, we addressed the possibility of systematic deviations originating from the use of single-spin- $1 / 2$ Bloch equations for modeling nutation profiles. While the anomeric proton in sucrose is decoupled from the covalently bonded carbon $\left({ }^{1} \mathrm{~J}_{\mathrm{CH}}=169 \mathrm{~Hz}\right)$ during $t_{n u t}$ through the use of a $4 \mathrm{kHz}$ DIPSI-2 composite pulse decoupling scheme, the anomeric carbon is also scalar coupled to the proton on the neighbouring carbon that is off-resonance to

Table 1

A comparison of ${ }^{13} \mathrm{C}$ RF field amplitudes measured on a sucrose sample using the onresonance nutation experiment $\left(B_{1}\right.$ nutation $)$ and CONDENZ ( $B_{1}$ conDENZ). $B_{1}$ nutation values are reported to the same number of decimal places as the $B_{1}$ conDENZ values to facilitate comparison. $B_{1}$ inhomogeneities extracted from the CONDENZ profiles at each $B_{1}$ field are listed in column 3. (ND: not determined).

\begin{tabular}{llll}
\hline $\mathbf{B}_{\mathbf{1} \text { setting }}(\mathbf{H z})$ & $\mathbf{B}_{\mathbf{1} \text { CONDENZ }}(\mathbf{H z})$ & $\mathbf{I}_{\mathbf{B} \mathbf{1}}(\%)$ & $\mathbf{B}_{\mathbf{1}}$ nutation \\
\hline 1 & $0.98 \pm 0.01$ & $\mathrm{~Hz})$ \\
2 & $2.00 \pm 0.01$ & ND & 0.91 \\
3 & $2.95 \pm 0.01$ & $\mathrm{ND}$ & 2.04 \\
5 & $4.95 \pm 0.04$ & $\mathrm{ND}$ & 3.03 \\
7.5 & $7.47 \pm 0.02$ & $5.3 \pm 0.5$ & 5.03 \\
10 & $9.94 \pm 0.01$ & $5.0 \pm 0.2$ & 7.50 \\
15 & $14.98 \pm 0.02$ & $4.6 \pm 0.3$ & 14.99 \\
20 & $19.79 \pm 0.03$ & $4.2 \pm 0.2$ & 20.07 \\
25 & $24.99 \pm 0.03$ & $4.1 \pm 0.2$ & 24.98 \\
30 & $29.67 \pm 0.04$ & $4.6 \pm 0.3$ & 30.04 \\
100 & $97.20 \pm 0.04$ & $4.65 \pm 0.04$ & 96.30 \\
500 & $486.3 \pm 0.1$ & $5.42 \pm 0.05$ & 485.9 \\
750 & $731.6 \pm 0.2$ & $5.21 \pm 0.04$ & 731.1 \\
1000 & $973.4 \pm 0.4$ & $4.72 \pm 0.03$ & 975.6 \\
1500 & $1456.9 \pm 0.3$ & $5.16 \pm 0.04$ & 1465.0 \\
2000 & $1943 \pm 3$ & $6.0 \pm 0.1$ & 1965 \\
\hline
\end{tabular}

the DIPSI-2 field by $1.86 \mathrm{ppm}$, through a two-bond ${ }^{2} \mathrm{~J}_{\mathrm{CH}}$ of $7 \mathrm{~Hz}$ [59]. These two-bond and three-bond couplings could impact the fit $B_{1}$ values especially at small amplitudes of the RF field. In order to probe this possibility, we first simulated nutation profiles at various $B_{1}$ fields using product operators for an AMX spin system, where $A$ and $M$ are the anomeric carbon and proton $\left({ }^{1} \mathrm{~J}_{\mathrm{CH}}=169 \mathrm{~Hz}\right)$ that are on-resonance to the $\mathrm{B}_{1}$ and decoupling fields respectively, and $\mathrm{X}$ is the neighbouring proton that is $1.3 \mathrm{kHz}$ off-resonance to the decoupling field and scalar-coupled to the anomeric carbon with a ${ }^{2} \mathrm{~J}_{\mathrm{CH}}$ of $7 \mathrm{~Hz}$ and to the anomeric proton with a ${ }^{3} \mathrm{~J}_{\mathrm{HH}}$ of $4 \mathrm{~Hz}$. The simulated profiles were then fit with the single-spin$1 / 2$ equations above to extract the amplitude of the $B_{1}$ field (Fig. S2). The input and fit $B_{1}$ values correlate excellently $\left(R^{2}=0.99\right)$ and agree to within $0.1 \%$ over a range of $B_{1}$ values from $0.5-10 \mathrm{~Hz}$ (Fig. S2, Table S3), confirming that the use of the single-spin-1/2 equations for modeling the nutation profiles does not systematically distort the measured $B_{1}$ values.

\subsection{CONDENZ profiles provide robust estimates of the $R F$ inhomogeneity}

Having established the utility of our method for accurately and precisely measuring RF fields, we next asked whether the offsetdependent modulations observed in our experiments are sensitive to $B_{1}$ inhomogeneity. In order to address this question, we constructed $\chi_{\text {red }}^{2}$ curves that reflect the robustness of the parameter estimates of $\mathrm{I}_{\mathrm{B} 1}$. These curves show pronounced minima for $\mathrm{B}_{1}>5 \mathrm{~Hz}$ (Fig. 5A, Fig. S1), confirming that $\mathrm{I}_{\mathrm{B} 1}$ can also be measured reliably with our method. The errors in $\mathrm{I}_{\mathrm{B} 1}$ range from $\sim 9 \%$ at $7.5 \mathrm{~Hz}$ to $<1 \%$ at $1 \mathrm{kHz}$ (Table 1 ), showing that the estimates are more precise at higher RF field strengths. For $\mathrm{B}_{1} \leq 5 \mathrm{~Hz}, \chi_{\text {red }}^{2}$ curves are often flat on the lower limit of $\mathrm{I}_{\mathrm{B} 1}$, suggesting that only an upper estimate can be extracted reliably (Fig. S1). We verified these conclusions by fitting data simulated using the procedure detailed above to Eq. (9). I $I_{B 1}$ values recovered from the fit match very well with the input values for $B_{1} \geq 5 \mathrm{~Hz}$ (Table S3), while there are systematic differences between $0.1-5.5 \%$ for $B_{1}<5 \mathrm{~Hz}$.

\subsection{Estimating ${ }^{15} \mathrm{~N} R \mathrm{R}$ amplitudes using CONDENZ data}

Since $R_{1 \rho}$ and CEST data are acquired primarily on ${ }^{13} \mathrm{C}$ and ${ }^{15} \mathrm{~N}$ nuclei [36], we next explored the possibility of measuring ${ }^{15} \mathrm{~N} R \mathrm{RF}$ fields using the CONDENZ approach. We chose ${ }^{15} \mathrm{~N}^{\varepsilon}$-labeled Trp as a suitable small molecule because of a number of favorable properties such as easy availability, the slow solvent exchange rate of the indole ${ }^{1} \mathrm{H}^{\varepsilon}[60]$, a sharp indole ${ }^{1} \mathrm{H}^{\varepsilon_{-}}{ }^{15} \mathrm{~N}^{\varepsilon}$ correlation, and a ${ }^{15} \mathrm{~N}$ chemical shift that falls within the resonance frequency range of typical protein and nucleic acid molecules. In order to eliminate potential interference from $\mathrm{H} / \mathrm{D}$ exchange in the nutation profiles, we used $2.5 \% \mathrm{~d}_{6}$-DMSO as the lock solvent [47]. CONDENZ profiles 

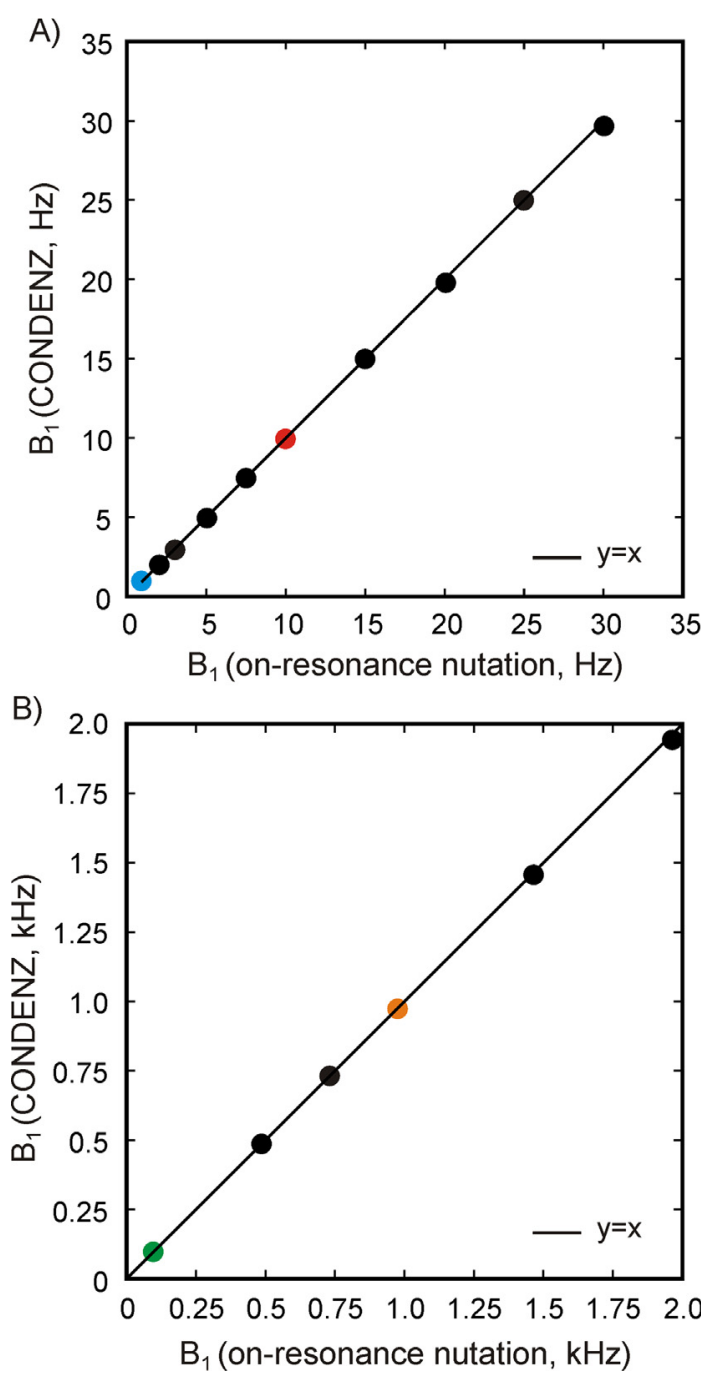

Fig. 4. A comparison of the $B_{1}$ field amplitude obtained by modeling CONDENZ profiles (y-axis) with the values determined from an on-resonance nutation experiment (x-axis) for weak (1-35 Hz, panel A) and strong (100-2000 Hz, panel B) $B_{1}$ fields. The solid line in both panels is a $y=x$ function. $B_{1}$ data points obtained by fitting CONDENZ profiles shown in Fig. 1 are indicated with the same colour scheme as in Fig. 1. Error bars are generally smaller than the size of the data points and not visible in the plot.

of the Trp indole ${ }^{15} \mathrm{~N}^{\varepsilon}$ nucleus also show offset-dependent modulations in the presence of a $B_{1}$ field similar to those seen for the sucrose anomeric ${ }^{13} \mathrm{C}$ (Fig. 6A, Fig. S3), confirming that these modulations are independent of the identity of the nucleus. Nutation profiles were modeled using the Bloch equations (Eq. (9)) to extract values of $B_{1}$ and $I_{B 1}$. Similar to ${ }^{13} C$, the amplitude of $B_{1}$ fields applied on the ${ }^{15} \mathrm{~N}$ channel also can be measured accurately and precisely with steep $\chi_{\text {red }}^{2}$ and small errors (0.05-0.5\%) (Fig. S3, Table S4) for $\mathrm{B}_{1}$ fields $\geq 2 \mathrm{~Hz}$, demonstrating the generality of the methodology.

\subsection{Practical aspects of using CONDENZ profiles to determine $B_{1}$ field strength}

There are a few practical considerations that govern the utilization of this methodology. First and foremost, the nutation parameters such as $t_{n u t}$ and the offset spacing $(\delta \Delta)$ must be adjusted so that the squared-sinc modulation is clearly observed in the CONDENZ profile. If $t_{n u t}$ or $\delta \Delta$ are too large, or if the signal-to-noise ratio (SNR) is too low so that clear modulations are not observed, the $B_{1}$ field cannot be extracted reliably. Parameters that provide tractable CONDENZ profiles and accurate $B_{1}$ fields in the range from $1-2000 \mathrm{~Hz}$ are listed in Table S1 and can be used as initial estimates, though we have observed that $t_{n u t}$ values may have to be slightly modified based on the $R_{2}$ of the observed nucleus; if the $R_{2}$ is higher than for the anomeric carbon of sucrose, smaller $t_{n u t}$ values can be employed to visualize the modulations clearly. Second, a vast number of CEST and $R_{1} \rho$ experiments are carried out on large biomolecules such as proteins and nucleic acids, where $R_{2}$ is too large to observe squared-sinc modulations. In such cases, we asked whether small molecules can be used as internal or external standards for measuring the $B_{1}$ field. First, we doped a ${ }^{15} \mathrm{~N}$-labeled ubiquitin sample with an internal ${ }^{15} \mathrm{~N}^{\varepsilon}$-Trp standard and measured the RF field amplitude for $B_{1}$ values ranging from $1-50 \mathrm{~Hz}$ using the on-resonance nutation experiment for ubiquitin and the CONDENZ approach for Trp (Fig. 6B). The values agree to within $1 \%$ and correlate with an $\mathrm{R}^{2}$ of 0.99 (Table S5) for $B_{1} \geq 2 \mathrm{~Hz}$, demonstrating that both molecules experience the same $B_{1}$ field. In contrast, there is a $13 \%$ difference between the $1 \mathrm{~Hz} \mathrm{~B} \mathrm{~B}_{1}$ fields measured using the on-resonance (ubiquitin) and CONDENZ approaches (Trp) (Table S5), highlighting the difficulty of locating the exact ${ }^{15} \mathrm{~N}$ chemical shift of Gln62 while acquiring on-resonance nutation data. Next, we made an external standard of ${ }^{15} \mathrm{~N}^{\varepsilon}$-Trp in the same buffer and measured the $\mathrm{B}_{1}$ field using CONDENZ. The magnitude of the RF field matches excellently with those estimated from the internal Trp standard (to within $4 \%$ ) as well as with ubiquitin, provided the tuning, matching, pulse widths and power levels are left unchanged between the analyte and the external standard (Table S5). These results unequivocally show that the CONDENZ approach for determining the RF amplitude can be used in conjunction with an external small molecule standard and extend its utility for sensitive biomolecular samples that may be affected by the addition of small molecule standards. Third, $R_{1 \rho}$ and CEST experiments on biomolecules span a wide range of resonance offsets [28,29,56,61-68]. Specifically, experiments on ${ }^{13} \mathrm{C}$ nuclei in proteins are acquired on moieties with chemical shifts from $5 \mathrm{ppm}\left({ }^{13} \mathrm{C} \delta\right.$ of Ile) to $175 \mathrm{ppm}$ (carbonyl carbons) that corresponds to $34 \mathrm{kHz}$ on an $800 \mathrm{MHz}$ spectrometer. In order to determine whether the same external standard (e.g. the anomeric ${ }^{13} \mathrm{C}$ of sucrose resonating at $92 \mathrm{ppm}$ ) can be used to estimate the $\mathrm{B}_{1}$ amplitude for such a wide range of ${ }^{13} \mathrm{C}$ transmitter frequencies, we prepared a sample containing a mixture of benzaldehyde, sucrose and $\alpha$-ketobutyric acid, and acquired CONDENZ profiles on the methyl- ${ }^{13} \mathrm{C}$ of ${ }^{13} \mathrm{CH}_{3} \alpha$-ketobutyric acid $(\varpi=6.5 \mathrm{ppm})$, the anomeric ${ }^{13} \mathrm{C}$ of sucrose $(\varpi=92 \mathrm{ppm})$, an aromatic ${ }^{13} \mathrm{C}$ of benzaldehyde $(\varpi=135.5 \mathrm{ppm})$ and the aldehydic ${ }^{13} \mathrm{C}$ of benzaldehyde $(\boldsymbol{\omega}=196.5 \mathrm{ppm})$. The magnitude of the $\mathrm{B}_{1}$ field determined with these four ${ }^{13} \mathrm{C}$ nuclei spanning a range of $\sim 200 \mathrm{ppm}$ in chemical shift agree to within a maximum deviation of $1.2 \%$ for $\mathrm{B}_{1}$ fields of $5 \mathrm{~Hz}$ or larger, while a deviation of $12 \%$ is observed for the $2 \mathrm{~Hz}$ case (Table S6). Though the deviations observed in the mixture sample are small, these experiments suggest that it is preferable to use a calibration standard whose resonance frequency matches well with the nucleus on which $R_{1 \rho}$ or CEST experiments are carried out, especially where small $(1-3 \mathrm{~Hz}) \mathrm{B}_{1}$ fields are involved. Finally, a SNR of the reference standard of 200 is sufficient to guarantee good quality CONDENZ profiles which can be analyzed to determine the amplitude of the RF field. For a $100 \mathrm{mM}$ unlabeled sucrose sample (natural abundance ${ }^{13} \mathrm{C}$ ) or $1 \mathrm{mM}{ }^{15} \mathrm{~N}^{\varepsilon}$-Trp, nutation data with such SNR can be obtained in $45 \mathrm{~min}$ on a $700 \mathrm{MHz}$ spectrometer equipped with a room-temperature probe, underscoring the accessibility and ease of application of our method. 

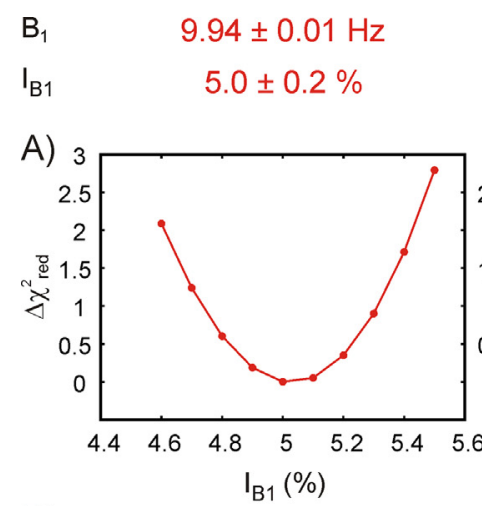

B)

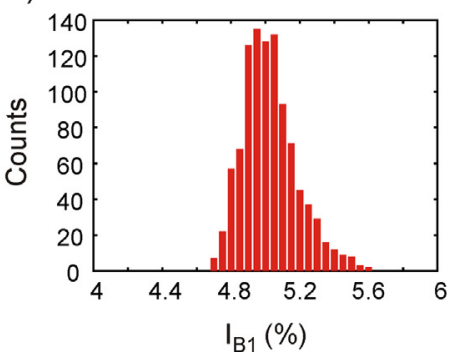

$24.99 \pm 0.03 \mathrm{~Hz}$

$4.1 \pm 0.2 \%$
$97.20 \pm 0.04 \mathrm{~Hz}$

$4.65 \pm 0.04 \%$
$973.4 \pm 0.4 \mathrm{~Hz}$

$4.72 \pm 0.03 \%$
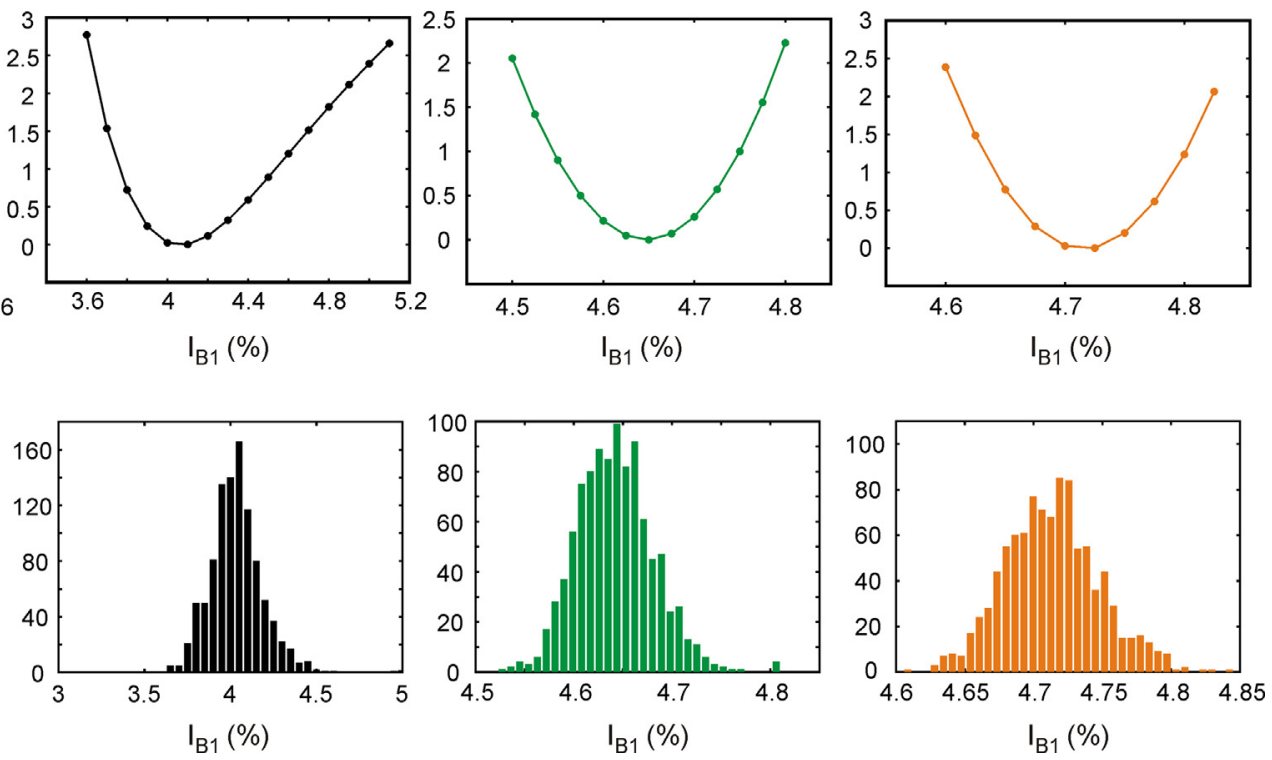

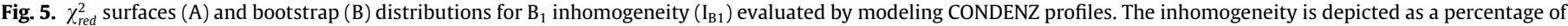

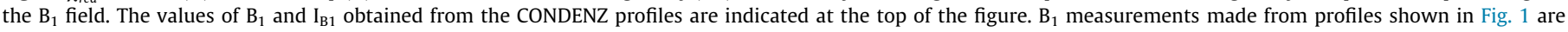
coloured with the same scheme.
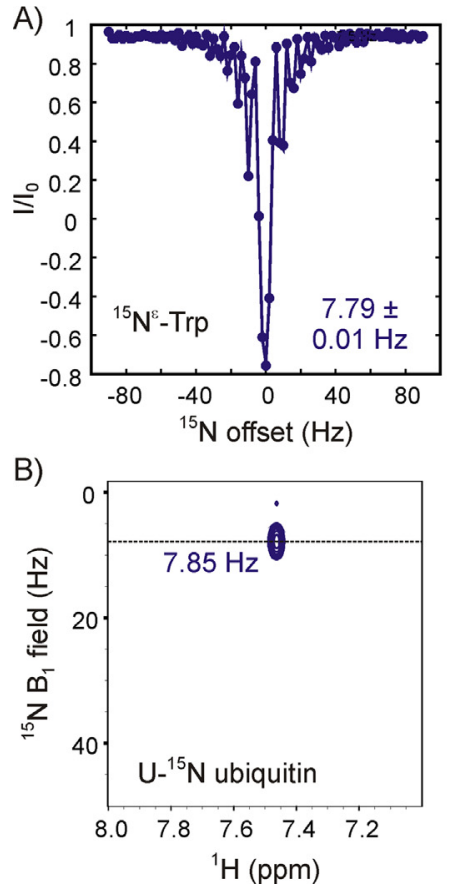
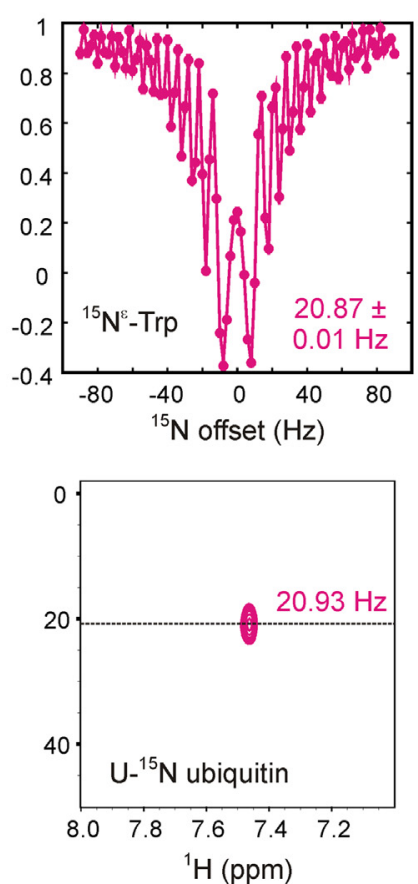

C)

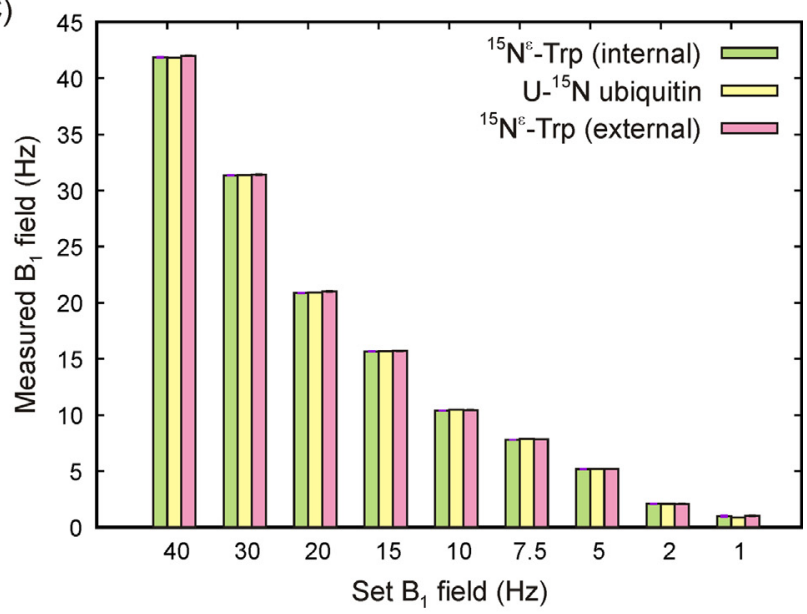

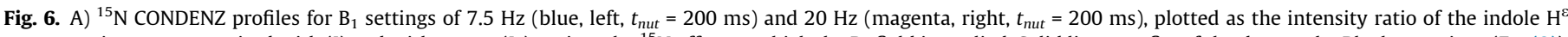

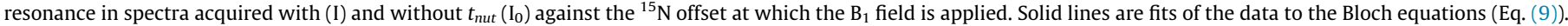

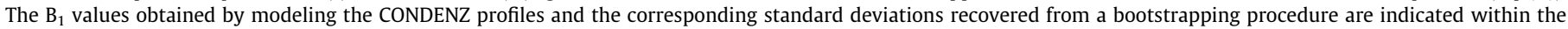

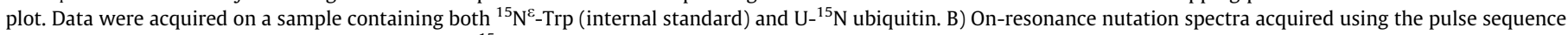

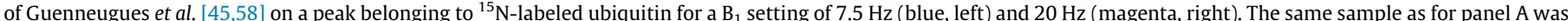

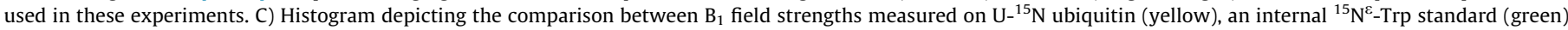

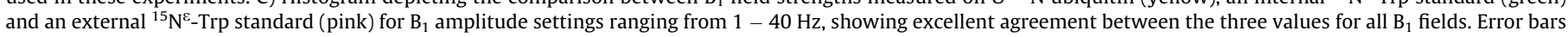

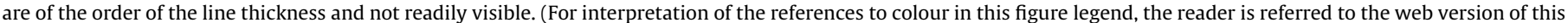
article.) 


\section{Conclusions}

The CONDENZ approach is particularly useful for measuring weak $B_{1}$ fields of the order of $1-10 \mathrm{~Hz}$ that are routinely employed in CEST experiments. In this $B_{1}$ regime, the on-resonance nutation method is susceptible to interference from off-resonance effects, because locating the resonance frequency to within a few $\mathrm{Hz}$ is difficult for biomolecules with $10 \mathrm{~Hz}$ or larger linewidths. The CONDENZ method, however, is immune to off-resonance effects as the approach relies on measurements made by varying the chemical shift offset. The chemical shift of the analyte nucleus that is used for calibration is a fitting parameter and therefore does not have to be exactly identified. In addition, our experiments demonstrate that $\mathrm{B}_{1}$ calibration can be carried out with a small molecule external standard, eliminating the necessity for finding an isolated protein or nucleic acid resonance that does not undergo conformational exchange for this purpose. We anticipate such flexibility to be particularly useful for large biomolecules and intrinsically disordered proteins, whose 2D correlation spectra are characterized by severe peak overlap.

\section{Declaration of Competing Interest}

The author declare that there is no conflict of interest.

\section{Acknowledgments}

We thank Dr. Tairan Yuwen for help with the simulations for the AMX spin system as well as Dr. Pramodh Vallurupalli and Dr. Siddhartha Sarma for a critical reading of the manuscript. This work was supported by the DBT/Wellcome Trust India Alliance Fellowship (grant no.: IA/I/18/1/503614) and a DST/SERB Core Research grant (no. CRG/2019/003457), as well as a start-up grant from IISc awarded to A.S. We also acknowledge funding for infrastructural support from the following programs of the Government of India: DST-FIST, UGC-CAS, and the DBT-IISc partnership program. C.N.V. thanks the Ministry of Human Resource Development, Government of India for fellowship support through the Prime Minister's Research Fellows scheme. A.J. thanks IISc Bangalore for fellowship support.

\section{Appendix A. Supplementary material}

Figures showing CONDENZ profiles for sucrose and Trp for all RF field strengths, simulations showing CONDENZ profiles for an AMX spin system, table detailing the fitted $B_{1}$ and $I_{B 1}$ values for Trp, a comparison between input $\mathrm{B}_{1}$ field strength in the AMX simulation and the fit value assuming a single-spin approximation, tabulated values of RF amplitudes obtained with ubiquitin as well as internal and external small molecule standards, and a comparison of RF amplitudes obtained with different small molecule probes. Supplementary data to this article can be found online at https://doi.org/ 10.1016/j.jmr.2021.107032.

\section{References}

[1] T.R. Alderson, L.E. Kay, NMR spectroscopy captures the essential role of dynamics in regulating biomolecular function, Cell 184 (3) (2021) 577-595.

[2] A. Sekhar, L.E. Kay, An NMR view of protein dynamics in health and disease, Annu. Rev. Biophys. 48 (2019) 297-319.

[3] R. Schneider, M. Blackledge, M.R. Jensen, Elucidating binding mechanisms and dynamics of intrinsically disordered protein complexes using NMR spectroscopy, Curr. Opin. Struct. Biol. 54 (2019) 10-18.

[4] B. Liu, H. Shi, H.M. Al-Hashimi, Developments in solution-state NMR yield broader and deeper views of the dynamic ensembles of nucleic acids, Curr. Opin. Struct. Biol. 70 (2021) 16-25.

[5] S.-R. Tzeng, C.G. Kalodimos, The role of slow and fast protein motions in allosteric interactions, Biophys. Rev. 7 (2) (2015) 251-255.
[6] L. Deshmukh, V. Tugarinov, D.H. Appella, G.M. Clore, Targeting a dark excited state of HIV-1 nucleocapsid by antiretroviral thioesters revealed by NMR spectroscopy, Angew. Chem. Int. Ed. 57 (10) (2018) 2687-2691.

[7] G.P. Lisi, J.P. Loria, Solution NMR spectroscopy for the study of enzyme allostery, Chem. Rev. 116 (11) (2016) 6323-6369.

[8] A.G. Palmer III, Chemical exchange in biomacromolecules: past, present, and future, J. Magn. Reson. 241 (2014) 3-17.

[9] J.P. Loria, M. Rance, A.G. Palmer, A relaxation-compensated Carr-PurcellMeiboom-Gill sequence for characterizing chemical exchange by NMR spectroscopy, J Am Chem Soc 121 (10) (1999) 2331-2332.

[10] F.A. Mulder, N.R. Skrynnikov, B. Hon, F.W. Dahlquist, L.E. Kay, Measurement of slow ( $\mu s-m s$ ) time scale dynamics in protein side chains by $15 \mathrm{~N}$ relaxation dispersion NMR spectroscopy: application to Asn and Gln residues in a cavity mutant of T4 lysozyme, J. Am. Chem. Soc. 123 (5) (2001) 967-975.

[11] P. Vallurupalli, A. Sekhar, T. Yuwen, L.E. Kay, Probing conformational dynamics in biomolecules via chemical exchange saturation transfer: a primer, J. Biomol. NMR 67 (4) (2017) 243-271.

[12] N.L. Fawzi, J. Ying, R. Ghirlando, D.A. Torchia, G.M. Clore, Atomic-resolution dynamics on the surface of amyloid-[bgr] protofibrils probed by solution NMR, Nature 480 (7376) (2011) 268-272.

[13] S.K. Whittier, A.C. Hengge, J.P. Loria, Conformational motions regulate phosphoryl transfer in related protein tyrosine phosphatases, Science 341 (6148) (2013) 899-903.

[14] I.J. Kimsey et al., Dynamic basis for dG• dT misincorporation via tautomerization and ionization, Nature 554 (7691) (2018) 195-201.

[15] G. Bouvignies et al., Solution structure of a minor and transiently formed state of a T4 lysozyme mutant, Nature 477 (7362) (2011) 111-114.

[16] X. Yao, M.K. Rosen, K.H. Gardner, Estimation of the available free energy in a LOV2-Ja photoswitch, Nat. Chem. Biol. 4 (8) (2008) 491-497.

[17] B. Zhao, S.L. Guffy, B. Williams, Q. Zhang, An excited state underlies gene regulation of a transcriptional riboswitch, Nat. Chem. Biol. 13 (9) (2017) 968974.

[18] M.R. Evans, P.B. Card, K.H. Gardner, ARNT PAS-B has a fragile native state structure with an alternative $\beta$-sheet register nearby in sequence space, Proc. Natl. Acad. Sci. 106 (8) (2009) 2617-2622.

[19] D.M. Korzhnev, T.L. Religa, W. Banachewicz, A.R. Fersht, L.E. Kay, A transient and low-populated protein-folding intermediate at atomic resolution, Science 329 (5997) (2010) 1312-1316.

[20] D.M. Korzhnev et al., Nonnative interactions in the FF domain folding pathway from an atomic resolution structure of a sparsely populated intermediate: an NMR relaxation dispersion study, J. Am. Chem. Soc. 133 (28) (2011) $10974-$ 10982.

[21] P. Neudecker et al., Structure of an intermediate state in protein folding and aggregation, Science 336 (6079) (2012) 362-366.

[22] E. Rennella, A. Sekhar, L.E. Kay, Self-assembly of human Profilin-1 detected by Carr-Purcell-Meiboom-Gill nuclear magnetic resonance (CPMG NMR) spectroscopy, Biochemistry (Mosc) 56 (5) (2017) 692-703.

[23] Sekhar A, et al. (2015) Thermal fluctuations of immature SOD1 lead to separate folding and misfolding pathways. eLife 4:e07296.

[24] A. Sekhar et al., Probing the free energy landscapes of ALS disease mutants of SOD1 by NMR spectroscopy, Proc. Natl. Acad. Sci. USA 113 (45) (2016) E6939E6945.

[25] T. Xie, T. Saleh, P. Rossi, C.G. Kalodimos, Conformational states dynamically populated by a kinase determine its function, Science 370 (6513) (2020).

[26] E.N. Nikolova et al., Transient Hoogsteen base pairs in canonical duplex DNA, Nature 470 (7335) (2011) 498-502.

[27] E.N. Nikolova, F.L. Gottardo, H.M. Al-Hashimi, Probing transient Hoogsteen hydrogen bonds in canonical duplex DNA using NMR relaxation dispersion and single-atom substitution, J. Am. Chem. Soc. 134 (8) (2012) $3667-$ 3670.

[28] I.J. Kimsey, K. Petzold, B. Sathyamoorthy, Z.W. Stein, H.M. Al-Hashimi, Visualizing transient Watson-Crick-like mispairs in DNA and RNA duplexes, Nature 519 (7543) (2015) 315.

[29] E.A. Dethoff, K. Petzold, J. Chugh, A. Casiano-Negroni, H.M. Al-Hashimi, Visualizing transient low-populated structures of RNA, Nature 491 (7426) (2012) 724-728.

[30] Sekhar A, et al. (2018) Conserved conformational selection mechanism of Hsp70 chaperone-substrate interactions. eLife 7:e32764.

[31] N. Lokesh, A. Seegerer, J. Hioe, R.M. Gschwind, Chemical exchange saturation transfer in chemical reactions: a mechanistic tool for NMR detection and characterization of transient intermediates, J Am Chem Soc 140 (5) (2018) $1855-1862$.

[32] C. Lorenz et al., The Structure of [HSi9] 3- in the Solid State and Its Unexpected Highly Dynamic Behavior in Solution, Angew Chem Int Ed 57 (39) (2018) 12956-12960.

[33] V. Ramanujam, C. Charlier, A. Bax, Observation and kinetic characterization of transient Schiff base intermediates by CEST NMR spectroscopy, Angew Chem Int Ed 58 (43) (2019) 15309-15312.

[34] J. Kim et al., Indirect detection of intermediate in decarboxylation reaction of phenylglyoxylic acid by hyperpolarized 13 C NMR, Chem Commun 56 (95) (2020) 15000-15003.

[35] S. Knecht et al., Indirect Detection of Short-Lived Hydride Intermediates of Iridium N-Heterocyclic Carbene Complexes via Chemical Exchange Saturation Transfer Spectroscopy, The Journal of Physical Chemistry C 123 (26) (2019) $16288-16293$. 
[36] A.G. Palmer, C.D. Kroenke, J.P. Loria, Nuclear magnetic resonance methods for quantifying microsecond-to-millisecond motions in biological macromolecules, Methods Enzymol 339 (2000) 204-238.

[37] G. Karunanithy, J. Reinstein, D.F. Hansen, Multiquantum Chemical Exchange Saturation Transfer NMR to Quantify Symmetrical Exchange: Application to Rotational Dynamics of the Guanidinium Group in Arginine Side Chains, The journal of physical chemistry letters 11 (14) (2020) 5649-5654.

[38] F. Bloch, Dynamical theory of nuclear induction, II. Phys Rev 102 (1) (1956) 104.

[39] W.A. Anderson, Nuclear magnetic resonance spectra of some hydrocarbons, Phys Rev 102 (1) (1956) 151.

[40] H. Torrey, Transient nutations in nuclear magnetic resonance, Phys Rev 76 (8) (1949) 1059.

[41] J. Leigh Jr, New technique for radio frequency magnetic field measurement, Rev Sci Instrum 39 (10) (1968) 1594-1595.

[42] R. Ernst, Nuclear magnetic double resonance with an incoherent radiofrequency field, J. Chem. Phys. 45 (10) (1966) 3845-3861.

[43] K. Pachler, Residual splittings in off resonance decoupled NMR spectra, J Magn Reson 7 (4) (1972) 442-443.

[44] F.A. Mulder, M. Akke, Carbonyl 13C transverse relaxation measurements to sample protein backbone dynamics, Magn Reson Chem 41 (10) (2003) 853865.

[45] M. Guenneugues, P. Berthault, H. Desvaux, A Method for DeterminingB1Field Inhomogeneity. Are the Biases Assumed in Heteronuclear Relaxation Experiments Usually Underestimated?, J Magn Reson 136 (1) (1999) 118-126

[46] Bax A (1981) Two-dimensional nuclear magnetic resonance in liquids.

[47] V.P. Tiwari, S. Pandit, P. Vallurupalli, Exchangeable deuterons introduce artifacts in amide $15 \mathrm{~N}$ CEST experiments used to study protein conformational exchange, J Biomol NMR 73 (1) (2019) 43-48.

[48] S.B. Azatian, N. Kaur, M.P. Latham, Increasing the buffering capacity of minimal media leads to higher protein yield, J Biomol NMR 73 (1) (2019) 11-17.

[49] C.M. Pickart, S. Raasi, Controlled synthesis of polyubiquitin chains, Methods Enzymol 399 (2005) 21-36.

[50] P. Pelupessy, E. Chiarparin, G. Bodenhausen, Excitation of selected proton signals in NMR of isotopically labeled macromolecules, (Elsevier) (1999).

[51] P. Pelupessy, E. Chiarparin, Hartmann-Hahn polarization transfer in liquids: an ideal tool for selective experiments, Concepts in Magnetic Resonance 12 (3) (2000) 103-124.

[52] S.P. Rucker, A. Shaka, Broadband homonuclear cross polarization in 2D NMR using DIPSI-2, Mol Phys 68 (2) (1989) 509-517.

[53] D.M. Korzhnev, V.Y. Orekhov, L.E. Kay, Off-resonance R1 $\rho$ NMR studies of exchange dynamics in proteins with low spin-lock fields: an application to a Fyn SH3 domain, J Am Chem Soc 127 (2) (2005) 713-721.
[54] A. Shaka, J. Keeler, T. Frenkiel, R. Freeman, An improved sequence for broadband decoupling: WALTZ-16, J Magn Reson 52 (2) (1983) 335-338.

[55] J. Cavanagh, W.J. Fairbrother, A.G. Palmer, N.J. Skelton, Protein NMR spectroscopy, Principles and Practice (Academic Press), 1995.

[56] P. Vallurupalli, G. Bouvignies, L.E. Kay, Studying "invisible" excited protein states in slow exchange with a major state conformation, J Am Chem Soc 134 (19) (2012) 8148-8161.

[57] B. Efron, R. Tibshirani, Bootstrap methods for standard errors, confidence intervals, and other measures of statistical accuracy. Stat Sci (1986) 54-75.

[58] T. Yuwen et al., Measuring solvent hydrogen exchange rates by multifrequency excitation 15N CEST: application to protein phase separation, J. Phys. Chem. B 122 (49) (2018) 11206-11217.

[59] K. Kato, T. Peters, NMR in Glycoscience and Glycotechnology, Royal Society of Chemistry.

[60] F. Kateb, P. Pelupessy, G. Bodenhausen, Measuring fast hydrogen exchange rates by NMR spectroscopy, J Magn Reson 184 (1) (2007) 108-113.

[61] A. Rangadurai, J. Kremser, H. Shi, C. Kreutz, H.M. Al-Hashimi, Direct evidence

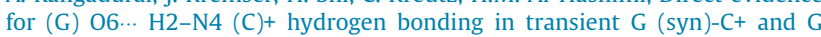
(syn)-m5C+ Hoogsteen base pairs in duplex DNA from cytosine amino nitrogen off-resonance R1 $\rho$ relaxation dispersion measurements, J Magn Reson 308 (2019) 106589.

[62] M.J. Grey et al., Characterizing a partially folded intermediate of the villin headpiece domain under non-denaturing conditions: contribution of His41 to the pH-dependent stability of the N-terminal subdomain, J Mol Biol 355 (5) (2006) 1078-1094.

[63] A.L. Hansen, E.N. Nikolova, A. Casiano-Negroni, H.M. Al-Hashimi, Extending the range of microsecond-to-millisecond chemical exchange detected in labeled and unlabeled nucleic acids by selective carbon R1 $\rho$ NMR spectroscopy, J Am Chem Soc 131 (11) (2009) 3818-3819.

[64] J.T. Baisden, J.A. Boyer, B. Zhao, S.M. Hammond, Q. Zhang, Visualizing a protonated RNA state that modulates microRNA-21 maturation, Nat. Chem. Biol. 17 (1) (2021) 80-88.

[65] B. Zhao, J.T. Baisden, Q. Zhang, Probing excited conformational states of nucleic acids by nitrogen CEST NMR spectroscopy, J. Magn. Reson. 310 (2020) 106642.

[66] G. Bouvignies, P. Vallurupalli, L.E. Kay, Visualizing side chains of invisible protein conformers by solution NMR, J. Mol. Biol. 426 (3) (2014) 763-774.

[67] D. Long, A. Sekhar, L.E. Kay, Triple resonance-based $13 \mathrm{C} \alpha$ and $13 C \beta$ CEST experiments for studies of ms timescale dynamics in proteins, J Biomol NMR (2014) 1-6.

[68] A.L. Hansen, L.E. Kay, Measurement of histidine pKa values and tautomer populations in invisible protein states, Proc. Natl. Acad. Sci. 111 (17) (2014) E1705-E1712. 